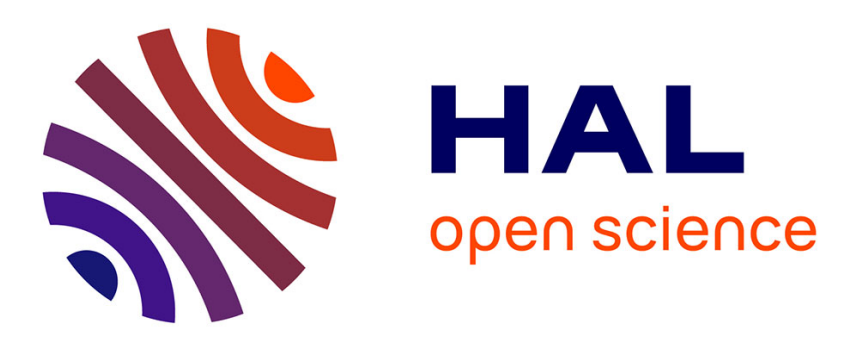

\title{
Le virus de l'enroulement des feuilles du cerisier, largement répandu en France sur noyer, est-il à l'origine de l'incompatibilité de greffage du noyer Juglans regia sur Juglans nigra?
}

René Delbos, Alain Bonnet, Jean Dunez

\section{To cite this version:}

René Delbos, Alain Bonnet, Jean Dunez. Le virus de l'enroulement des feuilles du cerisier, largement répandu en France sur noyer, est-il à l'origine de l'incompatibilité de greffage du noyer Juglans regia sur Juglans nigra?. Agronomie, 1984, 4 (4), pp.333-339. hal-00884642

\section{HAL Id: hal-00884642 \\ https://hal.science/hal-00884642}

Submitted on 1 Jan 1984

HAL is a multi-disciplinary open access archive for the deposit and dissemination of scientific research documents, whether they are published or not. The documents may come from teaching and research institutions in France or abroad, or from public or private research centers.
L'archive ouverte pluridisciplinaire HAL, est destinée au dépôt et à la diffusion de documents scientifiques de niveau recherche, publiés ou non, émanant des établissements d'enseignement et de recherche français ou étrangers, des laboratoires publics ou privés. 


\section{Le virus de l'enroulement des feuilles du cerisier, large- ment répandu en France sur noyer, est-il à l'origine de l'incompatibilité de greffage du noyer Juglans regia sur Juglans nigra?}

René DELBOS, Alain BONNET \& Jean DUNEZ

I.N.R.A., Station de Pathologie végétale, Centre de Recherches de Bordeaux, F 33140 Pont de la Maye

Sur les 2 millions de noyers exploités en France, la moitié environ est cultivée en verger : 20 p. 100 des arbres du verger français de noyer sont greffés sur Juglans nigra, le reste étant greffé sur $J$. regia. Des symptômes de nécrose de la zone de greffe sont signalés assez couramment sur les arbres greffés sur $J$. nigra, surtout depuis quelques années; ces symptômes sont tout à fait semblables à ceux décrits aux U.S.A. dans le cas d'arbres greffés sur $J$. hindsii ou "Paradox » (J. hindsii $\times J$. regia $)$. Le virus de l'enroulement chlorotique des feuilles du cerisier (cherry leaf roll : CLRV a été isolé de nombreux arbres présentant ce type de symptôme; le virus a été également isolé de noyers greffés sur J. regia et ne présentant aucun symptôme. D'une façon générale, le virus semble bien établi sur noyer en France. Les souches de CLRv isolées de noyer en France sont très proches, sinon identiques, aux souches de $C_{L R V}$ américaines. Une souche française BL 1 a été purifiée et plus spécialement étudiée : elle se présente comme une souche typique de CLRV (symptomatologie, nucléoprotéines, acides nucléiques). Un sérum a été préparé ; il est utilisé pour la détection du CLRV en verger ou en pépinière par le test ElisA.

La détection du virus se heurte à une difficulté résultant de la très irrégulière distribution du virus dans les tissus de $J$. regia lorsque celui-ci est greffé sur $J$. nigra ; il s'agit d'une distribution apparemment aléatoire ne résultant en aucun cas d'une diffusion lente du virus à partir d'un point d'infection. Lorsque les arbres sont greffés sur $J$. regia, le virus est réparti très uniformément dans les tissus de $J$. regia.

Le porte-greffe J. nigra n'est pas totalement résistant au virus : bien que le CLRV n'ait pu être isolé des parties aériennes, il a pu, dans ce cas, être mis en évidence dans les racines.

Il a été, à ce jour, impossible de détecter le CLRV chez un certain nombre d'arbres, $J$. regia/J. nigra présentant un net symptôme de nécrose de la ligne de greffe ; par ailleurs, différents auteurs ont isolé le CLRV d'arbres présentant, outre ce symptôme de nécrose, différents symptômes foliaires. La réussite des rétroinoculations de différentes souches purifiées de CLRV sur de jeunes semis de noyer devrait permettre d'établir de façon claire et définitive le rôle du CLRV dans ces faciès maladifs et de poursuivre avec précision les études de sensibilité variétale et de comportement des porte-greffes hybrides $J$. regia $\times J$. nigra.

Mots clés additionnels : Arbres fruitiers, purification, sérologie, ELISA, diagnostic.

Twenty per cent of the walnut trees cultivated in France in orchards are grafted on Juglans nigra. This rootstock was introduced about 20 years ago and the first disorders at the union were reported in the last 5 years. The disease observed in France is very similar to the black-line disease described in the U.S.A. on trees grafted on $J$. hindsii or "Paradox" ( $J$. hindsii $\times J$. regia). Cherry leaf roll virus was detected both in $J$. regia/J. nigra trees with black line and in $J$. regia/J. regia trees without any symptom and, generally speaking, the virus seems to be well established in walnuts in France.

The ClRV strains identified in France are very similar (if not identical) to the American walnut strains. One French strain, BL 1, from $J$. regia/J. nigra was purified and thoroughly investigated : it had all the characteristics of a typical CLRV strain. An antiserum was prepared and used for the routine detection of CLRV in orchards and nurseries using the ELISA technique. The main difficulty observed in the detection of CLRV arose from the uneven distribution of the virus in $J$. regia when this species is grafted on $J$. nigra: the virus seems to be randomly distributed in the tree and the restriction does not result from a slow diffusion of the virus in the plant from the infection points. In contrast, the virus is quite uniformly distributed in $J$. regia when this species is grafted on $J$. regia. This complicates indexing of CLRV when the tree is grafted on $J$. nigra and makes the test unreliable (sometimes, less than 5 per cent of the samples from an infected tree appear to be infected).

In a few trees showing typical black-line symptoms, we have not yet been able to identify CLRV; moreover some authors have described clear leaf symptoms that we have only observed on one tree in France. These two 
results pose the problem of the role of CLRV in the incompatibility of $J$, regia/J. nigra. The recent success of back inoculations of purified CLRV strains will allow us to answer this question and to get clear information on the susceptibility and behaviour of different rootstocks including some new $(J$. nigra $\times J$. regia) hybrids..

Additional key words : Fruit-trees, purification, serology, ELISA, diagnosis.

\section{INTRODUCTION}

Le semis de noix fut pendant longtemps le seul procédé utilisé pour la multiplication du noyer. Le perfectionnement progressif, depuis la fin du XIXe siècle, des techniques de greffage, a rendu possible la culture des variétés les plus intéressantes sur des porte-greffes adaptés. Jusqu'à une époque assez récente, le seul porte-greffe utilisé était le noyer anglais, Juglans regia L., de semis. Depuis une cinquantaine d'années, d'autres espèces de Juglans ont été utilisées comme porte-greffes : c'est le cas de Juglans hindsii Rehd. et de "Paradox " (J. hindsii $\times J$. regia) aux U.S.A., de Juglans nigra L. en Europe.

Il existe en France environ 2 millions de noyers dont la moitié croît sous forme d'arbres isolés; la seconde moitié est exploitée en vergers dans lesquels 80 à 85 p. 100 des arbres sont greffés sur $J$. regia, les 15 à 20 p. 100 restants étant greffés sur $J$. nigra. L'intérêt de ce dernier porte-greffe, utilisé depuis une vingtaine d'années, est son effet légèrement nanisant, une mise à fruits avancée de 4 à 5 ans par rapport au noyer anglais.$J$ regia et une bonne résistance à Phytophthora cinnamomi Rands. et Armillariella mellea (Fr.) Karst. Si les arbres greffés sur J. nigra représentent donc en moyenne 20 p. 100 du verger français, le chiffre varie, selon les régions, de $5 \mathrm{p} .100$ en Isère à $90 \mathrm{p} .100$ dans la vallée de la Garonne.

Dans les 5 dernières années, un symptôme de nécrose de la ligne de greffe analogue au «black line " décrit aux U.S.A. (MIRCETICH et al., 1980) est apparu dans les combinaisons $J$. regia/J. nigra et a été assez fréquemment observé au cours de prospections réalisées en Isère et en Périgord par la Station d'Arboriculture fruitière I.N.R.A. de Bordeaux et le Centre Technique Interprofessionnel des Fruits et Légumes (CTIFL). Il ne s'agit pas, en fait, de la première manifestation de ce type de désordre puisqu'en 1954 , GARAVEL avait déjà signalé des noyers $J$. regia greffés sur $J$. nigra qui présentaient un dépérissement très rapide à l'àge de 20-25 ans. L'auteur suggérait alors, comme origine de la maladie, une incompatibilité d'ordre génétique ; cette affection ressemble, par ailleurs, beaucoup à la maladie du « black line » connue aux U.S.A. en Oregon depuis 1924, bien décrite dès 1933 (SCHUSTER \& MilleR) dans le cas de $J$. regia/J. hindsii et pour laquelle différentes causes non infectieuses ont été avancées.

En 1976, SAvino et al. isolèrent en Italie, à partir de noyers présentant différents types de décolorations foliaires, deux virus à particules isométriques dont ils démontrèrent, un an plus tard (SAVINO et al., 1977), qu'ils appartenaient au groupe des Nepovirus et étaient, en particulier, reliés au virus de l'enroulement des feuilles de cerisier (cherry leaf roel virus : CLRV), virus qui est bien connu pour sa capacité à infecter de nombreuses espèces fruitières, forestières et herbacées (CROPLEY \& TOMLINSON, 1971 ; COOPER \& ATKINSON, 1975 ; JONES, 1976). En 1980, MIRCETICH et al. démontrèrent la présence du CLRV chez des noyers $J$. regia greffés sur $J$. hindsii et "Paradox" et présentant un symptôme de "black line ». En 1982, KOLBER \& NEMETH, en Hongrie, isolèrent également le CLRV à partir d'arbres greffés sur $J$. nigra et montrant, outre un symptôme de "black line ", différents symptômes foliaires très nets (taches, anneaux et lignes chlorotiques), très rarement décrits auparavant quel que soit le portegreffe utilisé. En 1982 également, DeLBos et al. ont isolé en France le CLRV de différents noyers greffés aussi bien sur $J$. nigra que sur $J$. regia. A côté du symptôme de nécrose de la ligne de greffe dans le cas d'arbres greffés sur $J$. nigra, peu de symptômes foliaires et peu de modifications de croissance ont pu être observés, quel que soit le porte-greffe.

Dès lors, un certain nombre de questions se trouvaient posées auxquelles a essayé de répondre le travail présenté ici. La première d'entre elles est une estimation du taux d'infection des vergers et des pépinières : le CLRV paraissant chez le noyer, comme chez d'autres essences, transmis assez rapidement par le pollen, un taux élevé de contamination rendrait son éradication impossible et risquerait donc d'interdire l'utilisation d'un porte-greffe comme $J$. nigra impliqué dans les symptômes d'incompatibilité. La seconde question est de confirmer nettement la responsabilité du CLRV ou de certaines souches de CLRV dans le symptôme d'incompatibilité observé chez J. regia/J. nigra et, ultérieurement, de déterminer le comportement de nouveaux porte-greffes hybrides $J$. regia $\times J$. nigra en cas d'infection par ce virus.

\section{MATÉRIELS ET MÉTHODES}

\section{A. Matériels}

Plusieurs souches de CLRV ont été utilisées notamment une souche issue de sureau (golden elderberry), une souche de l'American Type Culture Collection (ATCC: PV 166) et la souche CLRV noyer (W) fournie par S. M. MIRCETICH.

Une souche française a été isolée d'un noyer $J$. regia var. "Franquette " greffé sur $J$. nigra par transmission mécanique sur Chenopodium quinoa Willd. Elle a été purifiée sur cet hôte par 5 transferts de lésions locales à dilution limite et nommée BL 1 . 
Elle a été propagée sur différents hôtes (C. quinoa, C. foetidum Schrid., Nicotiana tabacum L., Petunia hybrida Hort., Pisum sativum L., Phaseolus vulgaris L.) et purifiée à partir de $C$. quinoa maintenus en serre à $20^{\circ} \mathrm{C}$. Une autre souche a été isolée de $J$. regia $/ J$. regia : elle a été dénommée $\mathrm{D}$ et ne présente apparemment aucune différence biologique ou sérologique avec la souche BL 1.

\section{B. Méthodes}

\section{Purification du virus}

Le CLRV est purifié à partir de feuilles de $C$. quinoa infectées, récoltées $10 \mathrm{j}$ après inoculation des plants et conservées à $-20^{\circ} \mathrm{C}$. La technique utilisée dérive de celle décrite par Doz et al. en 1981.

Le matériel végétal est broyé dans un tampon constitué de phosphate disodique-monopotassique $0,05 \mathrm{M}$, additionné d'acide ascorbique à $0,01 \mathrm{M}$, en présence de butanol-chloroforme $(\mathrm{w}: \mathrm{v}: \mathrm{v}=1: 3: 0,5)$ et ajusté à différents $\mathrm{pH}(5,5 ; 6,0 ; 7,0)$.

La phase aqueuse est récupérée et centrifugée $20 \mathrm{mn}$ à 10000 tours $/ \mathrm{mn}$. Le surnageant est additionné de 8 p. 100 de PEG 6000 , soumis à une agitation lente pendant $30 \mathrm{mn}$, puis laissé au repos $2 \mathrm{~h}$ à $4{ }^{\circ} \mathrm{C}$. La suspension est ensuite centrifugée $20 \mathrm{mn}$ à $6500 \mathrm{tr} / \mathrm{mn}$ (rotor Beckman JA 14). Les culots sont remis en suspension dans un tampon phosphate $0,05 \mathrm{M} \mathrm{pH} \mathrm{7,0} \mathrm{:} \mathrm{la} \mathrm{suspension} \mathrm{est} \mathrm{agitée} 10 \mathrm{mn}$ puis centrifugée $10 \mathrm{mn}$ à $10000 \mathrm{tr} / \mathrm{mn}$ (rotor Beckman JA 17). Le surnageant est récupéré puis ultracentrifugé $2 \mathrm{~h}$ à $40000 \mathrm{tr} / \mathrm{mn}$ (rotor Beckman $60 \mathrm{Ti}$ ). Le culot est remis en suspension dans du tampon phosphate $0,05 \mathrm{M}$ et la purification est poursuivie par une ultracentrifugation en gradient de saccharose (100-400 g/1 de tampon phosphate) pendant 2 h 30 à $37000 \mathrm{t} / \mathrm{mn}$ (rotor Beckman SW $41 \mathrm{Ti}$ ). Les fractions contenant le virus sont récupérées et concentrées par ultracentrifugation $2 \mathrm{~h}$ à $45000 \mathrm{tr} / \mathrm{mn}$ (rotor Beckman $60 \mathrm{Ti}$ ). Les culots sont remis en suspension dans le formaldéhyde à 0,2 p. 100 pour la préparation de sérums et les études immunologiques ou, pour l'extraction des acides nucléiques, dans un tampon Tris $0,01 \mathrm{M}, \mathrm{NaCl} 0,05 \mathrm{M}$, acétate $\mathrm{Na} 0,01 \mathrm{M}$, SDS 2 p. 100 .

\section{Etude des acides nucléiques du virus}

Après traitement des nucléoprotéines virales par le lauryle sulfate de $\mathrm{Na}$ (SDS) à $2 \mathrm{p} .100,10 \mathrm{mn}$ à $65^{\circ} \mathrm{C}$, les acides nucléiques sont directement séparés par électrophorèse sur gels cylindriques de polyacrylamide $2,5 \mathrm{p}$. 100. Après une pré-électrophorèse de $1 \mathrm{~h}$, la solution contenant les acides nucléiques est déposée sur le gel et la migration électrophorétique poursuivie pendant $3 \mathrm{~h}$ sous une tension de 7 à 8 volts $/ \mathrm{cm}$ et une intensité de 5 à $7 \mathrm{~mA} /$ gel. Les gels sont analysés à $265 \mathrm{~nm}$ dans un densitomètre UV Joyce Loebl.

\section{Préparation d'antisérums anti $\mathrm{BL} 1$}

Deux antisérums contre la souche BL 1 ont été preparés à partir de lapin et de poule. Les animaux ont reçu une $1^{\text {re }}$ injection de $0,4 \mathrm{mg}$ de virus dans $1 \mathrm{ml}$ de formol à 0,2 p. 100 additionné de $1 \mathrm{ml}$ d'adjuvant complet de Freund. Les injections ultérieures (4 à une semaine d'intervalle puis 3 à 3 mois d'intervalle, soit une immunisation totale de 1 an environ) sont faites en présence d'adjuvant incomplet.

Le sérum obtenu à partir du sérum de lapin a un titre en double diffusion en milieu gélosé de $1: 2$ 048. Le sérum isolé à partir des jaunes d'œufs de poule n'a pas été titré avant isolement des anticorps.

Les anticorps ont été isolés du sérum de lapin par chromatographie d'affinité sur Sépharose protéine A et les IgY des jaunes d'œufs de poule purifiés par la méthode de POLSON et al. (1980) par relargage au PEG 6000.

\section{Conditions de réalisation des tests ELISA}

Les tests ELISA ont été réalisés en plaques de microtitration Dynatech microELISA M 129 B.

Les extraits à analyser sont obtenus par broyage de feuilles ou de racines de noyer dans le tampon PBSTween-PVP (CLARK \& ADAMS, 1977) additionné de sulfate de nicotine à 5 p. 100 ; ce dernier est omis quand les extraits sont issus de plantes hôtes herbacées.

Dans la technique « sandwich » (CLARK \& ADAMS, 1977) les plaques sont sensibilisées par des IgG de lapin à $1 \mu \mathrm{g} / \mathrm{ml}$ et le conjugué des IgG avec la phosphatase alcaline utilisé dilué au $1: 2000$.

Dans la technique HADAS-ELISA (BAR JOSEPH \& MALKINSON, 1980) les conditions sont les suivantes :

a) $1^{\text {er }}$ protocole

- Sensibilisation des plaques : IgG lapin antiClRV $1 \mu \mathrm{g} / \mathrm{ml} ; 3 \mathrm{~h}$ à $37{ }^{\circ} \mathrm{C}$.

- Extrait de plantes 1 nuit à $4{ }^{\circ} \mathrm{C}$.

- IgY poule anti-CLRV $5 \mu \mathrm{g} / \mathrm{ml} ; 3 \mathrm{~h}$ à $37{ }^{\circ} \mathrm{C}$.

- Conjugué chèvre anti-poule (Nordic) $1: 5000$; $3 \mathrm{~h}$ à $37^{\circ} \mathrm{C}$.

\section{b) $2^{e}$ protocole}

- Sensibilisation des plaques IgY poule $2 \mu \mathrm{g} / \mathrm{ml}$; $3 \mathrm{~h}$ à $37^{\circ} \mathrm{C}$.

- Extrait de plantes : 1 nuit à $4{ }^{\circ} \mathrm{C}$.

- IgG lapin anti-CLRV : $1 \mu \mathrm{g} / \mathrm{ml} 3 \mathrm{~h}$ à $37{ }^{\circ} \mathrm{C}$.

- Conjugué chèvre anti-lapin (Nordic : 1 : 5000 ; $3 \mathrm{~h}$ à $37^{\circ} \mathrm{C}$.

La lecture est faite $1 \mathrm{~h}$ après addition du substrat dans un lecteur Multiskan Titertek ou Biomérieux Lov 1 et les résultats fournis après soustraction des réactions obtenues avec un extrait sain.

\section{Rétroinoculation de différentes souches de CLRV sur noyer}

La démonstration du rôle direct du CLRV dans l'expression de l'incompatibilité $J$. regia/J. nigra nécessite la retransmission de souches purifiées de virus sur noyer. Cette opération a été réalisée sur des semis de $J$. regia issus de la germination de noix prélevées sur un arbre (greffé sur $J$. regia) contrôlé 2 années de suite pour vérifier l'absence de CLRV. Les noix ont été semées après $24 \mathrm{~h}$ de stratification dans l'eau : les semis ont été élevés en serre à $20^{\circ} \mathrm{C}$ et inoculés par 4 méthodes d'inoculation mettant en 
œuvre de 20 à 50 répétitions par souche de virus et par technique :

- Inoculation mécanique de jeunes semis (au stade 2 feuilles) avec un extrait de feuilles infectées de C. quinoa darı un milieu constitué de phosphate disodique-monopotassique $0,05 \mathrm{M}$ additionné d'acide ascorbique $0,01 \mathrm{M}$.

- Implantation sous l'écorce du semis de noyer (au stade 2 feuilles) d'un lambeau d'épiderme de C. quinoa infecté systémiquement.

- Greffe d'approche entre un plant de $C$. quinoa infecté systémiquement et un semis de noyer (stade 3 feuilles, hauteur : 15 à $20 \mathrm{~cm}$ environ). Le contact est maintenu par une bande adhésive pendant 3 à 4 semaines.

- Implantation sous l'écorce du semis de noyer d'un morceau de papier buvard imbibé d'un extrait brut de feuilles de $C$. quinoa infectées.

Quatre souches de CLRV ont été utilisées : BL 1, D, W et PV 166.

Après inoculation, les semis sont maintenus en serre pendant 3 mois puis placés en pépinière.

\section{RÉSULTATS}

\section{A. Purification du virus et des acides nucléiques}

La souche BL 1 purifiée à partir de $C$. quinoa présente les caractéristiques classiques du CLRV et notamment, après centrifugation en gradient de saccharose, 2 composants nucléoprotéiques $\mathrm{B}$ et $\mathrm{M}$ (fig. 1). Il est à noter que l'obtention du composant $M$ nécessite, tout au long des opérations de purification, l'utilisation de valeurs de $\mathrm{pH}$ inférieures à 6 , voire à 5,5 (fig. $1 \mathrm{~A}, \mathrm{~B}, \mathrm{C}$ ). Le rendement en virus est de l'ordre de $4 \mathrm{mg} / \mathrm{kg}$ de feuilles.

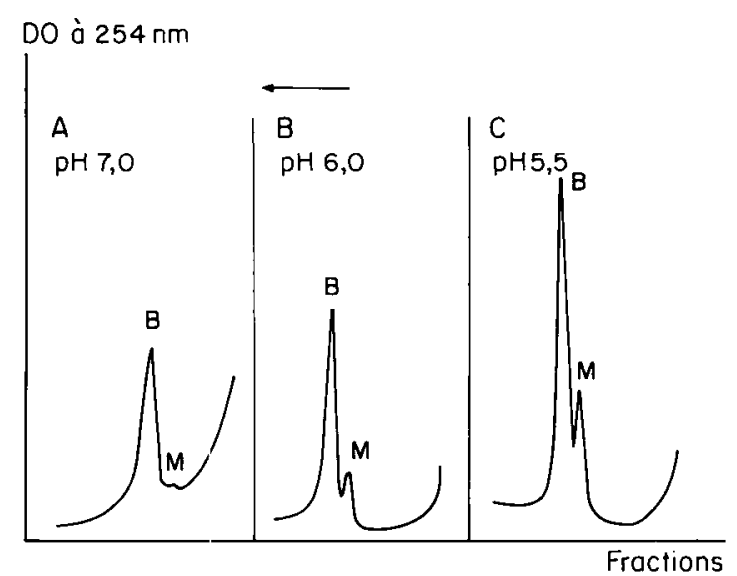

Figure 1

Profil d'absorption d'un gradient de saccharose $(100-400 \mathrm{~g} / \mathrm{l}$ tampon phosphate) après ultracentrifugation 2 h 30 à 37000 (Rotor Beckman SW 41 Ti) d'une suspension de CLRV:

$A-$ extraction et purification du virus à $\mathrm{pH} 7$

$B$ - extraction et purification du virus à $p H 6$

$C$ - extraction et purification du virus à $p H 5,5$

Absorption profile of sucrose gradient $1100-400 \mathrm{~g} / \mathrm{l}$ sucrose in phosphate buffer) ajter ultracentrifugation of a CIRV suspension for 2 h 30 at 37000 rpm (Rotor Beckman SW 41 Ti)

$A$ : extraction and purification carried out at $p H 7$

$B$ : extraction and purification carried out at $p H 6$

$C$ : extraction and purification carried out at pH 5.5
Le génome viral comporte classiquement 2 acides nucléiques infectieux de 2,1 et $2,4 \mathrm{~d}$ (fig. 2). L'existence de petits RNA chez le virus du groupe des Nepovirus étant fréquente, une recherche systématique de RNA de masse moléculaire comprise entre 0,1 et $0,5 \times 10^{6} \mathrm{~d}$ a été entreprise par électrophorèse sur gels de polyacrylamide à 5 p. 100 en plaques mais n'a montré aucune molécule de cette taille.

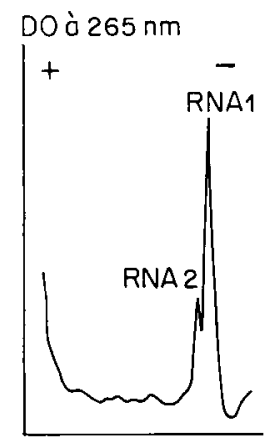

Figure 2

Profil d'absorption d'un gel de polyacrylamide à 2,5 p. 100 après électrophorèse d'une solution des acides nucléiques extraits d'une solution de $C_{L R V}$ purifiée d̀ pH 5,5; pré-électrophorèse $1 \mathrm{~h}$, 7 volts $/ \mathrm{cm}, 5 \mathrm{~mA} / \mathrm{gel}$; électrophorèse $3 \mathrm{~h}, 7$ volts $/ \mathrm{cm}, 5 \mathrm{~mA} / \mathrm{gel}$. Absorption of a cylindrical $2.5 \%$ polyacrylamide gel after electrophoresis of the RNAs extracted from CLRV particles purified at pH 5.5 ; pre-electrophoresis $1 \mathrm{~h}, 7 \mathrm{~V} / \mathrm{cm}, 5 \mathrm{~mA} / \mathrm{gel}$; electrophoresis $3 \mathrm{~h}, 7 \mathrm{~V} / \mathrm{cm}, 5 \mathrm{~mA} / \mathrm{gel}$.

\section{B. Application de la technique Elisa au diagnostic du CLRV}

Des investigations préalables (DELBOS et al., 1982) utilisant la technique ELISA ont démontré une très étroite parenté entre les souches BL 1, D, W, WVY (Walnut Vein Yellow) et WrS (Walnut Ring Spot, SAviNo et al., 1976). Tous les résultats mentionnés ici ont été obtenus avec le sérum anti BL 1 .

La technique ELISA sandwich détecte aisément $1 \mathrm{ng}$ de virus par ml de solution purifiée (fig. 3) et le CLRV est mis en évidence facilement tant à partir de plantes herbacées qu'à partir de feuilles de noyer (fig. 4).

Dans des études comparatives réalisées entre la technique ELISA normale et la technique HADAS cette dernière technique est apparue moins performante (fig. 4).

Tous les tests ont donc été réalisés par la technique ELISA sandwich en utilisant exclusivement des anticorps de lapin. Les valeurs des absorptions relevées avec les extraits sains ont été systématiquement soustraites des résultats. Elles ne sont jamais supérieures à 0,050 après $1 \mathrm{~h}$ d'incubation du substrat (fig. 4).

\section{Détection du ClRv en verger}

Le CLRV est présent chez les J. regia greffés sur 2 porte-greffes, $J$. nigra et $J$. regia, et aussi dans des arbres greffés sur $J$. hindsii (porte-greffe rarement utilisé en France).

Le virus a été isolé dans différentes régions de France (Corrèze, Dordogne, Lot, Gironde, Charente, Isère, Drome, etc...), chez de nombreuses variétés : 


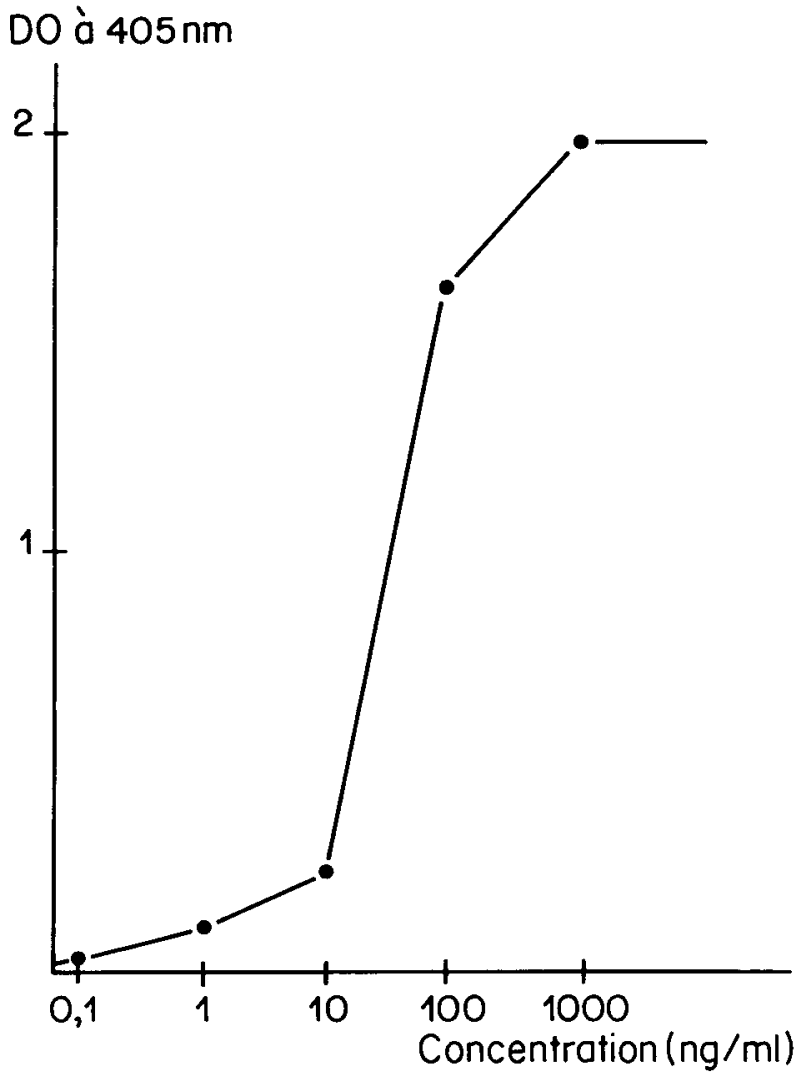

Figure 3

Analyse d'une suspension purifiée de CLRV par la technique ELISA. Analysis of a purified CLRV solution with the ELISA double antibody sandwich technique.

" Franquette », « Marbot », « Grosjean », « Parisienne », "Lozeronne », " Pedro » et « Hartley ». En revanche, il a été, malgré de nombreux essais, impossible de détecter le CLRV sur les parties aériennes de $J$. nigra quel que soit l'âge des arbres analysés. Néanmoins, dans quelques cas, les racines de $J$. nigra se sont révélées clairement porteuses de virus démontrant que cette espèce n'est pas totalement résistante au CLRV.

Le virus est très facile à mettre en évidence dans les tissus de $J$. regia, que l'arbre soit cultivé sur ses propres racines ou greffé sur $J$. regia; par contre, si l'arbre est greffé sur $J$. nigra, la distribution du virus dans les tissus de $J$. regia est extraordinairement irrégulière. Ces différences sont illustrées par quelques exemples de la recherche du virus chez des noyers de 15 ans environ sur chacun desquels de 20 à 80 échantillons ont ëté prélevés (tabl. 1). Lorsque l'arbre est greffé sur $J$. regia non seulement le virus est uniformément réparti mais la teneur en virus des échantillons, estimée par le test ELISA, est également homogène dans l'arbre ; à l'inverse, si le porte-greffe est un $J$. nigra, le virus n'est détecté que dans un petit nombre d'échantillons mais il y atteint une concentration analogue à celle observée dans le cas d'arbres infectés greffés sur $J$. regia; cette localisation très étroite du virus (les feuilles voisines paraissent, au test ElisA, totalement dépourvues de virus), semble aléatoire et n'évolue pas notoirement avec le stade de végétation ou avec le temps.

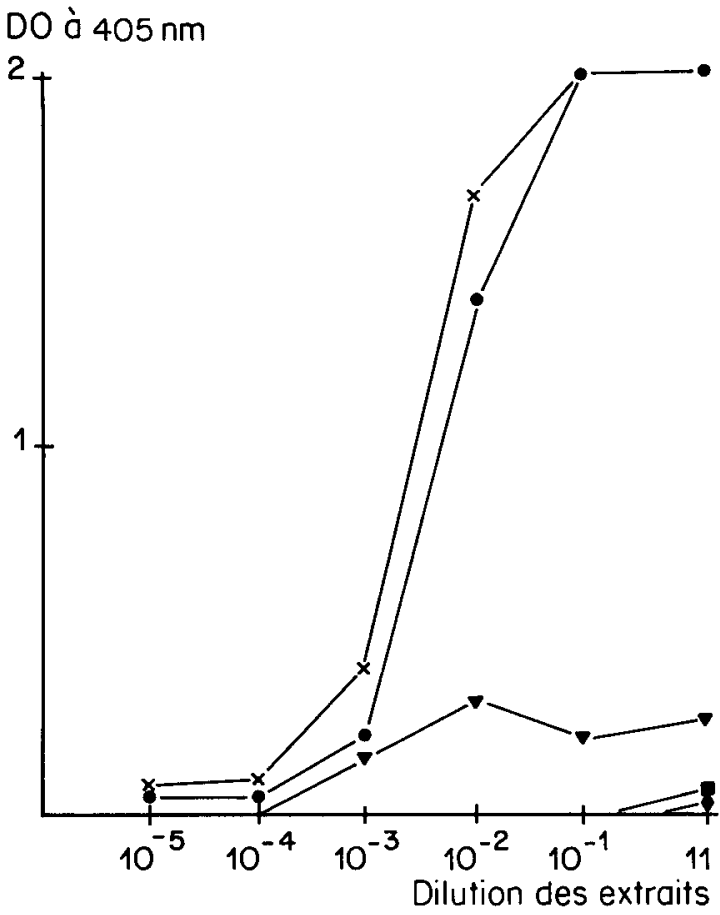

Figure 4

Détection du CLRV dans des feuilles de noyer ou de C. quinoa infectées. L'extrait a été préparé ( $w: v=1: 5)$ dans le tampon $P_{B S-P V P}$, additionné de sulfate de nicotine à 5 p. 100 dans le cas de feuilles de noyer.

Detection of $C_{L R V}$ in infected walnut or $C$. quinoa leaves. Extraction was carried out in PBS-PVP buffer $(w: v=1: 5)$ containing $5 \%$ nicotine sulfate when walnut leaves were used.

$\times-\times$ feuilles de $\mathrm{C}$. quinoa (technique sandwich) C. quinoa leaves (sandwich technique)

$\rightarrow$ feuilles de C. quinoa (technique HADAS) C. quinoa leaves (Hadas technique)

- - feuilles de J. regia (technique sandwich) J. regia leaves (sandwich technique)

- - extrait sain de C. quinoa (technique sandwich) C. quinoa healthy leaves (sandwich technique)

$\rightarrow$ extrait sain de J. regia (technique sandwich)

J. regia healthy leaves (sandwich technique)

Ces observations laissent actuellement 2 questions sans réponse : quelles sont, en cas d'arbres greffés sur $J$. nigra, les conditions à mettre en ouvre pour que les résultats d'un indexage appliqué en routine soient aussi fiables que possible ? Les quelques arbres présentant un symptôme de «black-line » et dans lesquels nous avons été incapables de déceler le CLRV sont-ils réellement dépourvus de virus?

D. Le ClRV est-il seul responsable des symptômes de nécrose de la ligne de greffe dans les combinaisons $J$. regia/J. nigra?

La réponse à cette question ne peut se trouver que dans le résultat de la rétroinoculation sur noyer de différentes souches de CLRV, préalablement isolées et purifiées.

Cette opération comporte 2 phases : la $1^{\text {re }}$ est la rétroinoculation, sur de jeunes semis de noyer, de suspensions de souches pures de virus issues de chénopode ; la $2^{\mathrm{e}}$ est, à partir des noyers ainsi infectés, la transmission en pépinière sur des combinaisons réputées capables d'extérioriser un symptôme de "black line » (J. regia greffé sur $J$. nigra, J. hindsii 
TABLEAU 1

Recherche du CIRv par le test ELISA dans des noyers de 15 ans environ (nombre d'échantillons infectés/nombre d'échantillons prélevés). Tous les échantillons sont constitués de feuilles de J. regia sauf pour l'échantillon A17 où feuilles de J. regia et racines de J. nigra ont été analysées.

Search for CLR.V infection by ELISA in 15-year old walnut trees (number infected/number of samples tested). All samples consisted of $\mathrm{J}$. regia leaves except A17 sample for which both leaves and roots were tested.

\begin{tabular}{|c|c|c|c|c|c|c|c|c|c|c|c|}
\hline \multirow{2}{*}{$\begin{array}{c}\text { Origine des échartillons : } \\
\text { Arbres étudiés : } \\
\text { variété/porte-greffe }\end{array}$} & \multirow{2}{*}{$\mathrm{BL}$} & \multicolumn{10}{|c|}{1982 Dates des prélèvements } \\
\hline & & 4 mai & $10 \mathrm{mai}$ & 22 mai & 28 mai & 9 juin & 20 juil. & 3 sept. & $\mathbf{1}^{\text {er }}$ juin & 8 juin & 29 juin \\
\hline (Marbot 99)/J. regia & - & $40 / 40$ & $40 / 40$ & 0 & 0 & 0 & 0 & 0 & 0 & 0 & 0 \\
\hline (Marbot 96$) / J$. regia & - & $33 / 33$ & $33 / 33$ & 0 & 0 & 0 & 0 & 0 & 0 & 0 & 0 \\
\hline $\begin{array}{l}\text { (Franquette A14) } / J \text {. nigra } \\
\text { (Franquette A17) } / J \text {. nigra }\end{array}$ & + & 0 & $6 / 59$ & $6 / 57$ & 0 & $6 / 51$ & $7 / 51$ & $4 / 51$ & $6 / 51$ & 0 & 0 \\
\hline feuilles & + & 0 & $1 / 36$ & $6 / 59$ & 0 & $4 / 60$ & $4 / 60$ & $1 / 42$ & $2 / 42$ & 0 & 0 \\
\hline racines & + & 0 & 0 & $1 / 1$ & 0 & $1 / 1$ & $1 / 1$ & $1 / 1$ & $5 / 5$ & 0 & 0 \\
\hline (Franquette $\mathrm{A} 1$ ) $/ J$. nigra & + & 0 & 0 & 0 & 0 & 0 & 0 & 0 & 0 & $1 / 83$ & $1 / 83$ \\
\hline (Franquette A2) $/ J$. nigra & + & 0 & 0 & 0 & 0 & 0 & 0 & 0 & 0 & $3 / 78$ & $2 / 90$ \\
\hline
\end{tabular}

,+- : Présence $(+)$ ou absence $(-)$ de nécrose de la ligne de greffe (Black line : BL) sur les arbres indexés. Presence $(+)$ or absence $(-)$ of black line at the union on tested trees.

0 : Test non réalisé. Not done.

TABLEAU 2

Résultats des rétroinoculations de souches purifiées de CLRV (maintenues chez Chenopodium quinoa) sur des semis de J. regia (nombre de semis infectés/nombre de semis inoculés).

Results of back inoculation of purified CLRV strains (propagated on Chenopodium quinoa) onto J. regia seedlings (number infected/ number of inoculated seedlings).

\begin{tabular}{|c|c|c|c|c|}
\hline \multirow[b]{2}{*}{ Souche } & \multicolumn{4}{|c|}{ Méthode d'inoculation } \\
\hline & Greffe d'approche & $\begin{array}{c}\text { Implantation d'un lambeau } \\
\text { d'épiderme de } \\
\text { C. quinoa infecté }\end{array}$ & $\begin{array}{c}\text { Implantation d'un morceau } \\
\text { de buvard imbibé } \\
\text { d'un extrait infecté }\end{array}$ & Inoculation mécanique \\
\hline BL 1 & $0 / 34$ & $0 / 32$ & $0 / 24$ & $7 / 24$ \\
\hline D & $0 / 18$ & $0 / 19$ & $0 / 48$ & $9 / 83$ \\
\hline W & $0 / 30$ & $0 / 25$ & $0 / 21$ & $7 / 20$ \\
\hline PV 166 & $0 / 32$ & $0 / 22$ & $0 / 28$ & $0 / 40$ \\
\hline Total & $0 / 114$ & $0 / 98$ & $0 / 121$ & $23 / 167$ \\
\hline
\end{tabular}

ou Paradox) dans un délai de 6 à 24 mois suivant l'inoculation (MIRCETICH et al., 1980). Seule jusqu'à présent la $1^{\text {re }}$ étape a été réalisée avec 4 souches de CLRV et 4 techniques d'inoculation. Les résultats en sont présentés au tableau 2 : les semis inoculés ont été l'objet de 2 contrôles : le $1^{\text {er }}$, fait un mois après l'inoculation, n'a montré aucune infection; le $2^{\mathrm{e}}$, 2 mois plus tard, a démontré que plusieurs semis avaient été infectés par les 2 souches françaises $D$ et $\mathrm{BL} 1$ et la souche américaine $W$. Les rétroinoculations réussies l'ont toutes été consécutivement à une inoculation mécanique. Les tentatives d'inoculation par «greffe » ou implantation subcorticale conduisent dans les tissus du noyer à un noircissement immédiat (vraisemblablement lié à des dépôts de tannins ou de mélanines) interdisant toute soudure, même temporaire, des tissus et constituant une barrière au passage du virus.

Le taux de réussite des infections par voie mécanique varie de 10 à 35 p. 100 selon les souches et il est possible que de nouveaux cas d'infection, non encore décelés, soient mis en évidence dans les mois qui vien- nent. Ce taux de transmission est, comparé aux résultats généralement observés dans les rétroinoculations de plantes herbacées à plantes ligneuses, remarquablement élevé : il devrait permettre très vite de conclure de façon définitive sur le rôle du CLRV dans le symptôme d'incompatibilité du $J$, regia/J. nigra.

\section{DISCUSSION}

Le CLRV, virus très polyphage et décrit chez de nombreuses espèces herbacées et ligneuses, est capable d'infecter $J$. regia ; bien que le virus ait été isolé aussi de racines de $J$. nigra, il n'a encore jamais été possible de le mettre en évidence dans les parties aériennes de cette espèce ce qui confirme son bon degré de résistance et rappelle les résultats décrits avec J. hindsii et "Paradox » (MIRCETICH et al., 1980). Le virus est présent en France sur des variétés greffées aussi bien sur $J$. regia que sur $J$. nigra. Différents auteurs en Italie (SAVINO et al., 1976) et en Hongrie (KOLBER \& NEMETH, 1982) ont décrit des 
symptômes foliaires de type taches annulaires dans le cas de nombreux arbres porteurs du CLRV. Dans toutes les investigations réalisées en France, un seul arbre $(J$. regia var « Marbot »/J. nigra) est apparu porteur de tels symptômes. Aucune corrélation ne semble donc pouvoir encore être faite entre la nécrose de la zone de greffe et ces symptômes foliaires dont l'origine demeure à préciser : souche de virus particulière, sensibilité variétale, conditions environnantes ou complexe de virus ? Compte tenu des succès que nous avons obtenus dans la phase initiale des rétroinoculations de différentes souches de CLRV sur noyer, le rôle exact de ce virus dans l'expression des symptômes devrait être rapidement établi.

De très nombreux arbres infectés ont été repérés : néanmoins, l'importance des contaminations des vergers par le CLRV est difficile à évaluer, essentiellement par suite des problèmes d'indexage posés, pour les arbres greffés sur $J$. nigra, par la distribution extraordinairement irrégulière et aléatoire du virus. Il semble peu probable que les quelques zones où le virus peut être isolé reflètent des points d'entrée dans la plante (lors de transmissions par le pollen par exemple) : d'une part, il est à peu près certain qu'une bonne proportion des arbres actuellement porteurs de CLRV étaient infectés à leur mise en place; d'autre part, on n'observe, d'une année sur l'autre, aucune diffusion significative du virus dans la plante et, peu de temps avant la mort de l'arbre, le virus demeure toujours extrêmement localisé. En outre, des portions infectées peuvent, en l'espace de quelques mois, apparaître dépourvues de virus en quantité décelable.

Les risques et les dégâts sont, semble-t-il, strictement liés aux arbres greffés sur $J$. nigra, aucun désor- dre n'ayant jamais été observé avec le porte-greffe $J$. regia; il importe de connaître au plus vite le comportement des nouveaux porte-greffes hybrides $J$. regia $\times J$. nigra qui seront probablement utilisés dans l'avenir pour les nouvelles variétés très productives en cours de sélection : pourront-ils à l'inverse de $J$. nigra être facilement infectés et manifesteront-ils un symptôme d'incompatibilité avec $J$. regia en cas d'infection?

De nombreux virus, très différents, sont impliqués dans des phénomènes d'incompatibilité ou des difficultés de greffage: dans chacun de ces cas, que l'incompatibilité apparaisse immédiatement après l'infection ou soit différée, l'effet du virus dépend des espèces et variétés en présence, des conditions environnantes, voire de la souche de virus. Il est difficile de savoir si le virus est directement la cause de l'incompatibilité ou si, plus vraisemblablement, il est un révélateur d'une médiocre compatibilité génétique. Quoi qu'il en soit, il importe que les protocoles de sélection sanitaire prennent en compte, pour les espèces greffées, non seulement les paramètres de sensibilité variétale appliqués séparément au greffon et au porte-greffe mais aussi l'effet du virus sur la compatibilité variété/porte-greffe.

Enfin, les mécanismes au travers desquels la présence d'un virus altère le fonctionnement de l'un ou de l'autre des composants en conduisant à une incompatibilité tissulaire et au rejet des tissus greffés forment, dans le domaine végétal, un champ d'investigations encore totalement vierge.

Recu le 18 août 1983. Accepté le 21 novembre 1983.

\section{RÉFÉRENCES BIBLIOGRAPHIQUES}

Bar Joseph M., Malkinson M., 1980. Hen egg yolk as a source of antiviral antibodies in the enzyme linked immunosorbent assay (ELISA) : a comparison of two plant viruses. J. Virol. Methods, 1 , 179-183.

Clark M. F., Adams A. N., 1977. Characteristics of the microplate method of enzyme linked immunosorbent assay for the detection of plant viruses. J. Gen. Virol., 34, 475-483.

Cooper J. I., Atkinson M. A., 1975. Cherry leaf roll virus causing a disease of Betula spp. in the United Kingdom. Forestry, 48, 193203.

Cropley R., Tomlinson J. A., 1971. Cherry leaf roll virus. C.M.I.A.A.B. Descr. Plant Viruses ${ }^{\circ} 80$.

Delbos R., Kerlan C., Dunez J., Lansac Micheline, Dosba Françoise, Germain E., 1982. Virus infection of walnuts in France. Acta Hortic., 130, 123-132.

Doz B., Macquaire G., Delbos R., Dunez J., 1981. Caractéristiques et rôle du RnA3, RNA satellite du virus des anneaux noirs de la Tomate. Ann. Virol. (Ann. Inst. Pasteur), 131 E, 489-499.

Garavel L., 1954. Enquête sur le comportement du noyer noir d'Amérique en tant que porte-greffe des variétés de noyers indigènes. Rev. For. Fr., 4, 69 p.
Jones A. T., 1976. Serological specificity of isolates of cherry leaf roll virus from different natural hosts. Poljopr. Znan. Smotra, 39/49, 527-532.

Kolber M., Nemeth Maria, 1982. Routine testing of English walnut mother trees and group testing of seeds by Elisa for detection of cherry leaf roll virus infection. Acta Hortic., 130, 161-162.

Mircetich S. M., Sanborn R. R., Ramos D. E., 1980. Natural spread, graft transmission and possible etiology of Walnut black line disease. Phytopathology, 70, 962-968.

Polson A., Von Wechmar M. B., Van Regenmortel M. H. V., 1980. Isolation of viral $\mathrm{IgY}$ antibodies from yolks of immunized hens. Immunol. Communic., 9, 475-493.

Savino B., Quacquarelli A., Gallitelli D., Piazzolla P., Martelli G. P., 1976. Occurrence of two sap transmissible viruses in walnut. Mitt. biol. Bundesanst. Land Forstwirtsch., H 170, 23-27.

Savino B., Quacquarelli A., Gallitelli D., Piazzola P., Martelli G. P., 1977. Il virus dell' accartocciamento fogliare del Ciliegio nel Noce. I. Identificazione e caratterizzazione. Phytopathol. Mediterr., 16, 96-102.

Schuster C. E., Miller P. W., 1933. A disorder of Person (English) walnuts grafted on black walnut stocks resulting in girdling. Phytopathology, 23, 408-409. 